\title{
Tenofovir Disoproxil Fumarate
}

National Cancer Institute

\section{Source}

National Cancer Institute. Tenofovir Disoproxil Fumarate. NCI Thesaurus. Code C47747.

A pro-drug, fumaric acid salt form of tenofovir, a nucleoside reverse transcriptase

inhibitor analog of adenosine. Tenofovir disoproxil fumarate is prescribed to treat HIV and chronic hepatitis B virus (HBV) in adults. 